\title{
Photometric properties of resolved and unresolved magnetic elements
}

\author{
S. Criscuoli ${ }^{1}$ and M. P. Rast ${ }^{2}$ \\ 1 Osservatorio astronomico di Roma, via Frascati 33, 00040 Monte Porzio Catone, Italy \\ e-mail: criscuoli@mporzio.astro.it \\ 2 Laboratory for Atmospheric and Space Physics, Department of Astrophysical and Planetary Science, University of Colorado, \\ Boulder CO, 80309-0391 USA \\ e-mail: mark.rast@lasp.colorado.edu
}

Received 20 June 2008 / Accepted 16 December 2008

\section{ABSTRACT}

\begin{abstract}
Aims. We investigate the photometric signature of magnetic flux tubes in the solar photosphere.
Methods. We developed two-dimensional, static numerical models of isolated and clustered magnetic flux tubes. We investigated the emergent intensity profiles at different lines-of-sight for various spatial resolutions and opacity models.

Results. We found that both geometric and photometric properties of bright magnetic features are determined not only by the physical properties of the tube and its surroundings, but also by the particularities of the observations, including the line/continuum formation height, the spatial resolution, and the image analysis techniques applied. We show that some observational results presented in the literature can be interpreted by considering bright magnetic features to be clusters of smaller elements, rather than a monolithic flux tube.
\end{abstract}

Key words. Sun: activity - Sun: photosphere - Sun: magnetic fields

\section{Introduction}

Recent observations taken in the G-band with sub-arcsecond resolution have allowed the study of the photometric (Berger et al. 2004; Okunev \& Kneer 2004; Ishikawa et al. 2007; Berger et al. 2007) and dynamic (Bovelet \& Wiehr 2003; Nisenson et al. 2003; Rouppe van der Voort et al. 2005; Ishikawa et al. 2007) properties of small-size magnetic-flux concentrations in the solar photosphere. Observations away from disk center have revealed that bright magnetic flux concentrations are usually accompanied by a dark lane on their disk-center side and by a bright tail on the limb side (Lites et al. 2004; Hirzberger \& Wiehr 2005; Berger et al. 2007). However, some bright points show no associated tail or dark lane, and some dark lanes with enhanced magnetic flux density show no associated bright point (Berger et al. 2007).

Somewhat contradictory measurements have been reported for the center-to-limb variation (CLV) of G-band bright point photometric contrast and size. For example Berger et al. (2007) reported maximum contrast at a position much closer to the limb than previous observers, while Hirzberger \& Wiehr (2005), after analysis of extreme limb observations, reported no trend in contrast or size with position on the solar disk. Discrepant CLVs have also been obtained when comparing G-band with other continua measurements (e.g. Auffret \& Muller 1991; Sütterlin 1999; Sánchez Cuberes et al. 2002; Okunev \& Kneer 2004). Together these observations have raised questions about the validity of viewing bright magnetic features as monolithic flux elements. In particular, it has been suggested that the observed CLVs of contrast and size are determined by properties of unresolved clusters of magnetic elements at the limb (e.g. Okunev \& Kneer 2005; Berger et al. 2007).
The properties of small individual magnetic features have been intensively investigated using both two and three dimensional magneto-hydrodynamic numerical simulations (e.g. Keller et al. 2004; Carlsson et al. 2004; Steiner 2005; Hasan et al. 2005; Uitenbroek \& Tritschler 2006). Some of the highlights of these modeling efforts include the following. Using both static and dynamic two-dimensional simulations, Steiner (2005) showed that the dark lane observed disk-center ward of bright magnetic elements is caused by the presence of cooler material adjacent to (both inside and outside) the disk-center ward flank of the magnetic tube. Similar results were previously presented by Pizzo et al. (1993a,b); Deinzer et al. (1984); Knölker et al. (1988a,b), who also investigated the sensitivity of the contrast intensity profiles to the physical properties of the tube (such as the temperature boundary condition, the evacuation of the tube, the intensity of magnetic field, and the inhibition of convection) and its temporal evolution. Ultimately, the intensity contrast profiles are determined by the degree of evacuation of the tube and the properties (particularly temperature) of the atmosphere inside and surrounding it. Fully three-dimensional magnetohydrodynamic simulations (e.g. Keller et al. 2004; Carlsson et al. 2004) have demonstrated that the increase in magnetic element contrast with distance from disk-center largely reflects the properties of the solar granulation behind the tube. This becomes visible away from disk-center because of the reduced opacity within the tube for highly oblique viewing angles. Moreover, De Pontieu et al. (2006), using both magneto-hydro-dynamic simulations and high spatial resolution observations, demonstrated that the temporal evolution of the photometric properties of magnetic flux tubes occurs on the characteristic granular time scale. 
The literature addressing the properties of flux tube aggregations is less extensive. Caccin \& Severino (1979) showed that the shape of the contrast-CLV of clusters of flux tubes is independent of the shape of the single underlying model tube (funnels or cylinders). More recently, Okunev \& Kneer (2005), following Karpinsky \& Okunev (1998), used three-dimensional static models in pressure equilibrium, to show that the appearance of aggregations of flux tubes and their measured polarimetric signals are strongly influenced by their position on the solar disk, filling factor and spatial resolution.

In this paper we investigate how the photometric signatures of isolated and clusters of small magnetic elements (such as the center-to-limb variation of photometric and geometric properties) reflect, not only the physical properties of the tube and its surroundings (such as temperature stratification and magnetic flux density), but also the particularities of the observation itself (such as spatial resolution, formation height of the wavelength of observation and image analyses technique employed). We examine these sensitivities using two-dimensional models of static magnetic flux tubes in radiative equilibrium with their surroundings. We show that results obtained by the analyses of recent high resolution observations can be interpreted assuming that observed magnetic bright features are actually aggregations of unresolved magnetic structures.

In Sect. 2 we describe the model. In Sect. 3 we investigate thermal properties of aggregations of magnetic flux tubes. In Sect. 4 we present the obtained contrast profiles and discuss their dependence on observational wavelength and spatial resolution. In Sects. 5 and 6 we show how the observed CLVs of photometric and geometric properties of aggregations of magnetic features depend not only on peculiarities of observations, but also on the numerical techniques employed to detect features on images. In Sect. 7 we investigate the size dependence of contrast. Conclusions are presented in Sect. 8.

\section{The model}

We consider static models of isolated and clustered magnetic flux tubes in Radiative Equilibrium (RE, hereafter) with the surrounding non-magnetic plane parallel atmosphere. The RE is imposed with an iterative scheme similar to the one proposed by Pizzo et al. (1993a) In brief, initial atmospheric conditions for the quiet sun and flux tube are imposed, the mean intensity everywhere in the domain is estimated and the logarithmic gradient $\nabla \equiv \mathrm{d} \ln T / \mathrm{d} \ln P$, where $T$ is the temperature and $P$ is the pressure, is calculated. A new temperature field is then computed imposing RE in those layers which do not satisfy the Schwarzschild criterion. A smooth variation of temperature between convectively stable and unstable layers is imposed using a linear interpolation. Namely, in the region $70 \mathrm{~km}$ thick where $\nabla \simeq \nabla_{\mathrm{ad}}$, the temperature is given by $T(x, z)=(1-\alpha) T(x, z)_{R}+\alpha T(x, z)_{C}$, where $T_{R}$ is the temperature value estimated by imposing RE, $T_{C}$ is the temperature value given by the assumed atmosphere model and $\alpha$ is a parameter which varies between 0 and 1. From the solution of the hydro-static and state (a perfect gas is assumed) equations the new pressure and density are computed respectively, while source function is estimated assuming local thermodynamic equilibrium (LTE). In this new atmosphere the mean intensity and the logarithmic gradient $\nabla$ are re-evaluated. The procedure is halted when temperature relative difference among two consecutive iterations is less than $0.001 \%$. The resulting atmosphere therefore differs from the initial one only in those layers which are stable against convection. The mean intensity is evaluated integrating over the solid angle by a Carlson
(Carlson 1963) quadrature scheme (namely, scheme A with 10 angles per octant). The intensity in each spatial direction is evaluated by a code (Criscuoli 2007) ${ }^{1}$ based on the shortcharacteristic technique (Kunasz \& Auer 1988). The initial non magnetic atmosphere is derived from the model of Kurucz (1994); the opacity is assumed to follow a power law in pressure and temperature. For a complete description of the atmosphere model see Giordano et al. (2007).

The presence of a flux tube is approximated by shifting a portion of the atmosphere downward prescribing the initial Wilson depression amplitude, which along with the size of the tube is a free parameter of the simulations. Note that, when iteration is halted, the final Wilson depression differs from the initial one. Incoming radiation at the top of the two-dimensional domain is set to zero while radiation at the bottom of the domain is estimated based on the radiative diffusion approximation. Horizontal periodicity is imposed at the vertical boundaries. The spatial domain is sampled by a grid with vertical and horizontal resolutions of $7.1 \mathrm{~km}$ and $3.5 \mathrm{~km}$, respectively. The vertical domain is $1130 \mathrm{~km}$ deep, $630 \mathrm{~km}$ above and $500 \mathrm{~km}$ below the optical depth unity. The horizontal extent of the domain is such that the ratio between the tube diameter (or of the cluster) and the width of the domain is large enough so that the presence of the tube does not significantly influence the temperature stratification of the quiet atmosphere (Fabiani-Bendicho et al. 1992).

The model solutions we present are thus based on the following assumptions. The geometry we consider is $2 \mathrm{D}$, although the radiative field and quadrature employed is fully $3 \mathrm{D}$. We expect this to reduce the effects of radiation channelling (see next section), although temperature differences between 2 and 3D calculations are estimated to be small (Uitenbroek 1998). Our model is also not in horizontal pressure equilibrium, which means that the tube geometry is simplified; flaring with height is not reproduced. As a consequence, the tube contrasts near the limb are somewhat reduced. However, thin flux tube models suggest that this flaring is negligible at photospheric heights we consider. Finally, our model assumes a gray opacity. Vögler (2004) showed that this assumption, by neglecting additional coupling of the radiation with matter (see for instance Steiner \& Stenflo 1990), leads to an under-estimation of the radiative illumination effect (see next section) in flux tubes, and therefore to underestimation of temperature in magnetic features. That author also showed that magnetic features contrast is under-estimated in gray calculations. We expect the same effects in results obtained with our calculations.

The models we developed are not meant to exactly reproduce the results obtained by recent high resolution observations, but rather to show qualitatively how measurements of contrast and geometric properties of isolated and clusters of magnetic elements are affected by observational issues.

\section{Thermal properties of clusters of flux tubes}

In this section we discuss the thermal properties of clusters of magnetic flux tubes, solutions in which more than one tube is in RE with the surrounding atmosphere. While we have investigated the thermal signatures of a range of flux tube sizes and configurations (i.e. number, strength, and separations), we present here only the results for aggregations of flux tubes with diameter $70 \mathrm{~km}$, center separation of $105 \mathrm{~km}$, and initial Wilson depression $150 \mathrm{~km}$.

${ }^{1}$ See http://dspace. uniroma2.it/dspace/handle/2108/599 

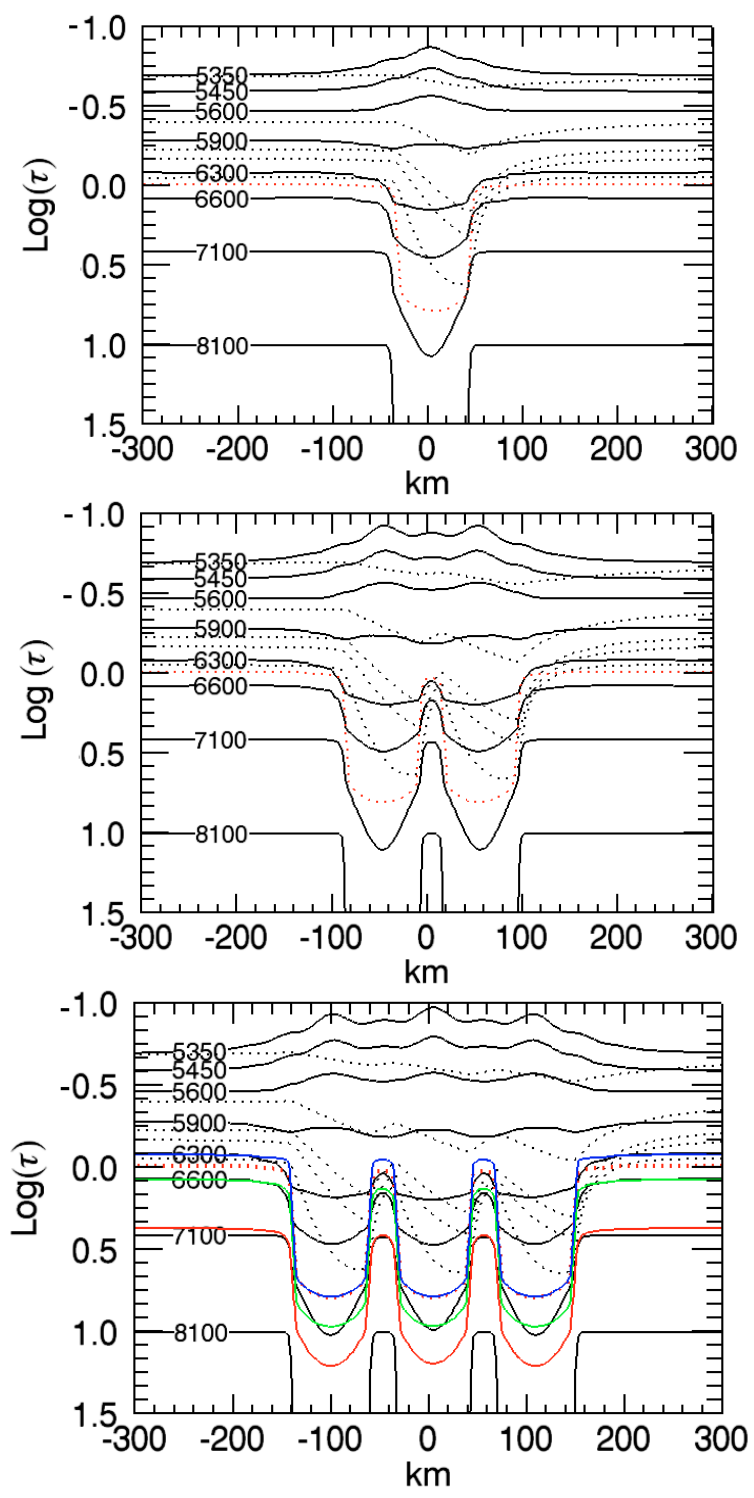

Fig. 1. Detail of the temperature field inside and around an isolated (top), two (center), three (bottom) magnetic flux tubes in RE. Diameters are $70 \mathrm{~km}$ and tubes axis are $105 \mathrm{~km}$ apart. The $x=0$ value of horizontal coordinate corresponds to the middle of the domain of the simulation. The continuous lines represent temperature iso-contours (in Kelvin). The dotted lines represent the heights at which $\tau=1$ for various linesof-sight: $\mu=0.2,0.4,0.6,0.7,0.9,1$ from top to bottom. The colored lines in the bottom panel represent the $\tau=1$ depth at $\mu=1$ for various opacity models. Red dotted line: gray model. Blue continuous line: $\lambda=8000 \AA$. Green continuous line: $\lambda=5000 \AA$. Red continuous line: $\lambda=16000 \AA$.

Examples of the temperature fields obtained by simulations are illustrated in Fig. 1. This shows the temperature field for an isolated (top), two (middle) and three (bottom) magnetic flux tubes. The final Wilson depression of the tubes in RE is approximately $60 \mathrm{~km}$ in all the models. Using the thin flux tube approximation, the magnetic field intensity along the tubes axis estimated at optical depth $\tau=1$ in the non magnetic atmosphere is approximately $1.5 \mathrm{kG}$ in all the configurations shown. The temperature at the bottom of the domain is lower then the surrounding atmosphere, as imposed by boundary and initial conditions. Because of the channeling (Cannon 1970; Kneer \& Trujillo-Bueno 1987; Fabiani-Bendicho et al. 1992; Hasan et al. 1999) of radiation escaping from the surrounding hotter

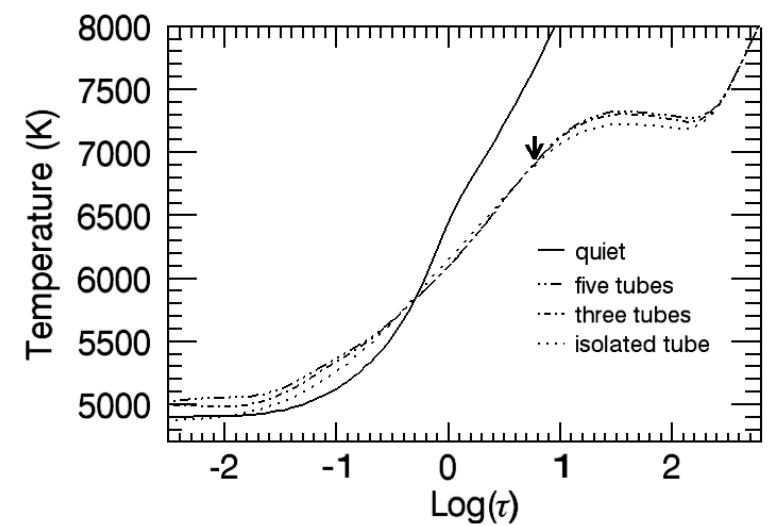

Fig. 2. Temperature along central axis versus optical depth for isolated, three and five tubes configurations in previous figures. The temperature profile of non magnetic atmosphere is given for reference. Arrow indicates optical depth unity along the axis of the isolated tube model.

atmosphere, the temperature difference between the magnetic elements and the non magnetic atmosphere decreases with decreasing optical depth. Eventually, in the optically thin part of the domain the temperature of the tubes and of an area in between and surrounding them exceeds the one of the non magnetic atmosphere. In particular, we note that between $0.5 \leq \tau \leq 2$ the temperature of plasma surrounding the tubes is lower with respect to the quiet atmosphere, while it increases at shallower depths. The depth at which the channeling is effective in heating the tubes, as well as the area of the surrounding atmosphere affected by the presence of the tubes, is determined by the ratio of the tube diameter and of the horizontal optical depth value (Pizzo et al. 1993b; Criscuoli 2007). The heating is larger along tubes flanks, and the effect is larger for external flanks of peripheral tubes (see for instance the isotherm at $6300 \mathrm{~K}$ in the three tubes case of Fig. 3).

Inspection of temperature fields also reveals that the amount of heating and cooling is a function of the number of flux tubes. This is better illustrated by the plot in Fig. 2, which shows the temperature variation with depth along the axis of an isolated tube and of the central tube of clusters of three and five elements. The temperature of the quiet atmosphere is given for reference. At heights $1<\log (\tau)<2$ heating is larger for aggregations of tubes with respect to the isolated one, but the increase in temperature is almost independent of the number of tubes in the cluster (three or five in this case). This is due to the fact that at these heights, because of the high value of the opacity (or of the horizontal optical depth), radiation escaping from the furthest tubes cannot penetrate into the central tube and contribute to its heating. On the contrary, at $\tau<1$ heating is largely dependent on the number of tubes. In these regions the increase in temperature is determined by the "illumination" from the layers at $\tau=1$ within and along the flanks of the tube (Pizzo et al. 1993b; Knölker et al. 1988a). The increase in the number of magnetic elements increases the width of the illuminating surface and therefore increases the temperature in the higher layers of the photosphere above the cluster. Between $0<\log (\tau)<1$ temperature is lower in clusters than near isolated tubes. There is a little dependence of this effect on the number of tubes. Note that a decrease of temperature in clusters of tubes was predicted by Deinzer et al. (1984).

Finally, we investigated the sensitivity of the temperature field to the increase in the number of adjacent flux tubes for various magnetic field intensities (for various values of the initial 

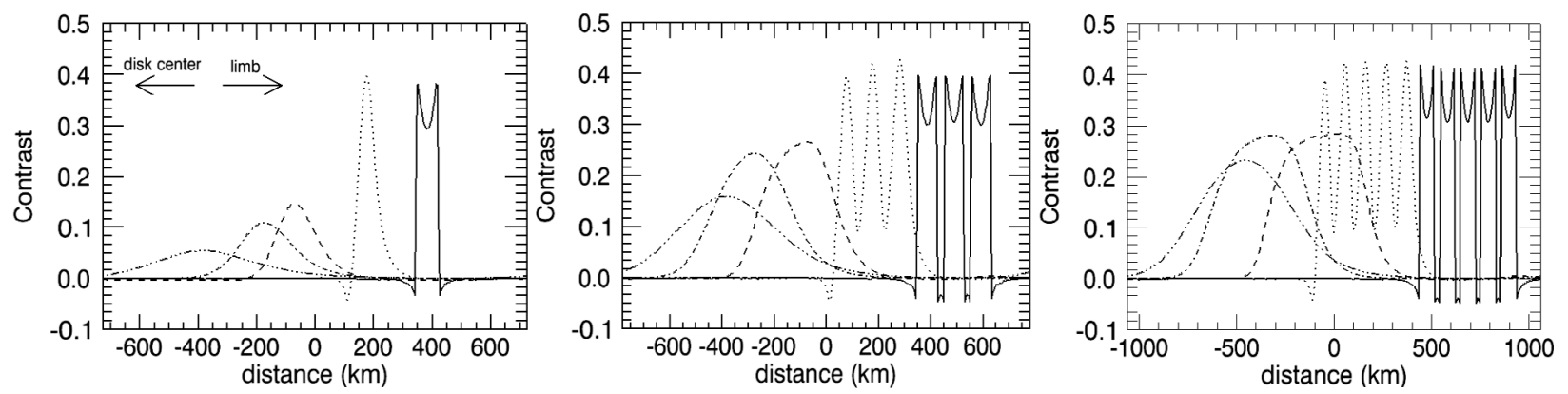

Fig. 3. Contrast profiles across an isolated, three and five flux tubes observed at different positions on the solar disk. Tubes are $70 \mathrm{~km}$ wide and separated (measured from tubes axis) by $105 \mathrm{~km}$. The disk center is on the left. Curves have been arbitrary shifted in order to fit into the same figure. Continuous line: $\mu=1$, dotted line: $\mu=0.9$; dashed line: $\mu=0.7$; dot-dashed line: $\mu=0.6$; dot-dot-dashed line: $\mu=0.4$.

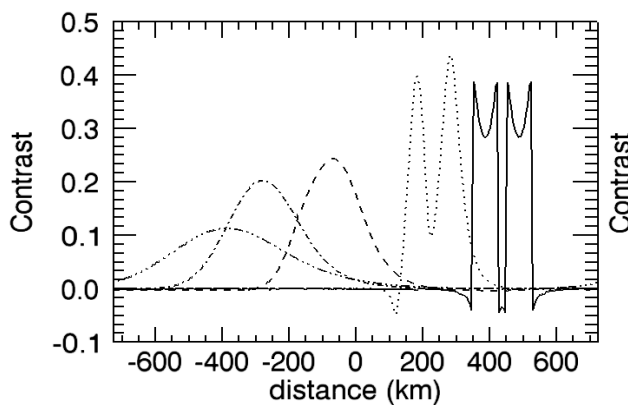

Fig. 4. Left: contrast profiles across two flux tubes $70 \mathrm{~km}$ wide $105 \mathrm{~km}$ apart. Right: contrast profiles of an isolated flux tube $175 \mathrm{~km}$ wide. Legend is as in Fig. 3.
Wilson depression). We found that, when holding the number of tubes constant, the stronger the magnetic field, the greater the increase in temperature at $\tau<1$ and the larger is the temperature decrease between $0<\log (\tau)<1$.

These results are in agreement with the ones previously obtained by Fabiani-Bendicho et al. (1992). Those authors investigated the dependence of temperature variations on filling factor by numerical 2D simulations, exploiting the horizontal periodicity of the intensity field and varying the horizontal size of the spatial domain of the simulations. Their models show the same temperature increase in the outer layers and decrease in lower layers of the atmosphere.

\section{Contrast profiles}

We define the contrast to be the ratio $I(x) / I_{q}-1$, where $I(x)$ is the emergent intensity value at each horizontal position $x$ and $I_{q}$ is the emergent intensity value far from the tube. We find, for various flux tube models and clusters configurations, that the contrast profiles depend on both the number of tubes and their separation distances. For example, Fig. 3 shows the contrast CLVs of an isolated flux tube and groups of two, three and five flux tubes of $70 \mathrm{~km}$ diameter and separation distance of $105 \mathrm{~km}$. At disk center, the profiles are characterized by two positive contrast "humps" and a surrounding negative contrast "ring". The "humps" are generated by the different inclinations of the $\tau=1$ contour with respect to the isotherms as shown in Fig. 1 (see also Deinzer et al. 1984; Knölker et al. 1988a,b). The "dark ring" is generated by the cool material surrounding the tube. We note that the maximum contrast value at $\mu=1$ slightly increases with the number of tubes. This reflects the slight increase in temperature with the number of adjacent tubes shown in Fig. 2 at the $\tau=1$ depth inside the tubes. Figure 3 also shows that the tubes are fully resolved only at disk center. The shallower the line-ofsight, the more the tubes appear as a single structure, with dimming on the disk-center side and brightening on the limb side, as observed for isolated flux tubes. This is in agreement with the findings of Okunev \& Kneer (2005), who showed that at the limb aggregations of simulated faculae appear as chains of small bright points. For lines-of-sight at which the tubes are still distinguishable, the ones which are closer to the limb appear brighter than the tubes closer to the disk. This is due to the fact that the radiation emitted at the limb side crosses more than one tube and is therefore less attenuated, or, in other words, the $\tau=1$ contour penetrates deeper into the atmosphere, thus sampling higher temperature layers, as also shown in Fig. 1. For similar reasons, the values of contrast at inclined lines-of-sight increase with the number of tubes in the cluster. Since for a given inclination and cluster configuration there is a finite number of tubes crossed by each ray, contrast increases with the number of tubes until this maximum number is reached. This maximum number increases with the inclination of the line-of-sight.

Figure 4 displays solutions for isolated and double flux tubes which occupy the same physical space: $175 \mathrm{~km}$. At disk center the contrast of the isolated large flux tube is lower with respect to the two small tubes case, while the contrary is observed off disk center. The opacity of the larger single flux tube is smaller than that of the two small tubes, and the $\tau=1$ contour penetrates deeper into the hotter non magnetic atmosphere. The consequent contrast profiles at different positions on the solar disk are quite different for the two configurations up to approximately $\mu=0.6$. In particular, we note that the disk-center ward dark lane is deeper and larger for the larger isolated flux tube than it is for the cluster. At smaller values of $\mu$ the contrast profiles of the two configurations are quite similar.

\subsection{Dependence on opacity model and spatial resolution}

We have computed the contrast profiles of isolated and clusters of flux tubes after varying the model opacity. This has allowed us to investigate the dependence of observed contrast profiles on the wavelength used for the observations. Continua opacities in three different wavelengths, 5000, 8000 and $16000 \AA$ A, were 


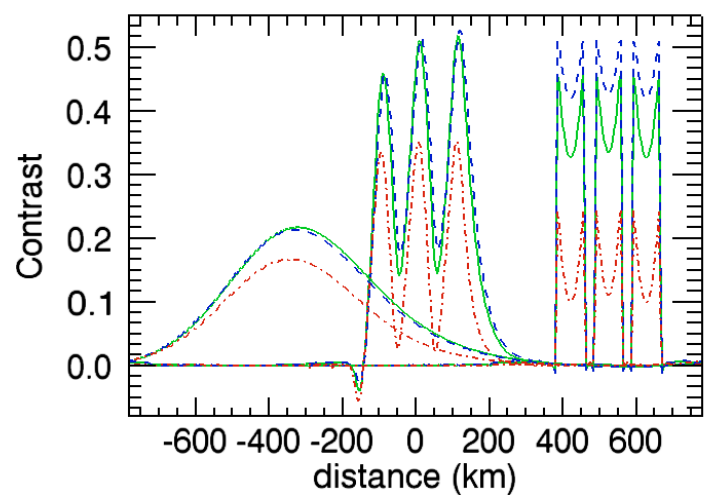

Fig. 5. Contrast profile across three tubes $70 \mathrm{~km}$ wide $105 \mathrm{~km}$ apart for various lines-of-sight and three different wavelengths. Blue, dot-dashed line: $\lambda=8000 \AA$. Green, continuous line: $\lambda=5000 \AA$. Red, dashed line: $\lambda=16000 \AA$. The disk center is on the left. From right to left: $\mu=1, \mu=0.9$ and $\mu=0.4$.

computed by using classical formula for continua processes and scattering of principal elements (see for instance Stix 2002). No blanketing line effects were considered. Abundances were taken from Grevesse et al. (1993).

We find that, because of the heating of the upper layers of the flux tubes, the contrast increases with the height of the $\tau=1$ depth at the wavelength being considered. The increase in contrast is larger at disk center, where larger variations of the Wilson depression occur, as shown for instance for the cluster of three tubes in Fig. 1. Figure 5 shows the corresponding contrast profiles at various lines-of-sight. We note that the negative contrast area surrounding the tubes at disk center, which is present for the corresponding gray atmosphere model illustrated in Fig. 3, is significantly reduced at the three wavelengths investigated. In fact, as shown in Fig. 1, in the grey model the $\tau=1$ line forms at a height at which the temperature of the plasma surrounding the tubes is lower with respect to the one of the non magnetic atmosphere. At wavelengths which form at larger and shallower depths the temperature differences between the area surrounding the tube and the quiet atmosphere are reduced and the dark ring is not observed. Variation with wavelength largely affects the contrast values for vertical lines-of-sight, as can be seen by comparison of contrast profiles illustrated in Fig. 5. In particular, the absolute contrast of dark lanes increases at wavelengths which sample the deepest layers (i.e. at optical grey depths larger than one), while they gradually disappear at wavelengths which sample shallower layers of the atmosphere (i.e. at optical grey depths smaller than one). Finally, the size of the bright area of the profiles (see next section) observed off disk center increases with the height of unity optical depth.

In order to investigate the effects of spatial resolution on observations, simulated contrast profiles have been projected onto the plane of the sky, that is the contrast profiles have been resized by the factor $\mu$, and convolved with Gaussian functions of different Full Width Half Maxima. We found that projection reduces the maximum contrast and the amplitude of the limb ward tail, thus producing smaller and more symmetric profiles. This is illustrated for instance by comparison of plots in Fig. 6 with those of Fig. 3. These show the contrast profiles of various flux tubes configurations after and before projection, respectively. Reduction in spatial resolution causes decrease of maximum contrast, broadening of profiles and smearing of small scale features, such as the dark lanes and the double "humps". These effects are larger for tubes observed at vertical lines-of-sight, and smaller for shallower lines-of-sight, where the intrinsic profiles are already blended by line-of-sight effects (Fig. 6). In particular, due to the presence of dark features, at vertical lines-of-sight the decrease of contrast values due to the reduction of spatial resolution is larger than at shallower lines-of-sights. As will be discussed in next section, these variations change the shape of the CLV. It is also interesting to note that in the models shown in Fig. 6 with resolution $0.1^{\prime \prime}$, the dark lane is present in the case of the isolated large flux tube, but is not present for clusters of small magnetic elements. Inspection of Fig. 6 reveals that the effects of reduction of resolution also depend on the number of flux tubes in a cluster. For instance, maximum contrast observed at disk center is approximately the same for the isolated, two and three tubes clusters at the resolution of the simulations. With the decrease of resolution, instead, a maximum contrast proportional to the flux tube number is observed for the three configurations.

\section{CLV of maximum and mean contrast}

We have estimated the CLV of maximum and mean contrasts of various flux tubes models and configurations. The mean contrast is defined as the average of contrast values larger than a specified threshold. Since for aggregations of flux tubes several distinct features can be selected in this way, we considered the average value of the contrast of each feature. Variations of the CLVs of mean contrast with variation of the threshold value were also investigated. Since the application of an intensity or contrast threshold is usually employed to detect features on images, this study gives an indication of the dependence of the measured contrast CLV on the identification method adopted.

We found that the CLV varies significantly with the number of flux tubes in a cluster. This is illustrated by Fig. 7, in which the CLV of maximum contrast, in the case of spatial resolution $0.1^{\prime \prime}$, is plotted for various models of isolated and clustered flux tubes. The plot shows that the curves flatten and the peak moves toward the limb as the number of flux tubes increases. The shift of the peak with an increase in filling factor was also found in 3-D simulations by Okunev \& Kneer (2005). In particular, it is interesting to note that the curve in Fig. 7 obtained for a cluster of five elements, resembles qualitatively and quantitatively the CLV obtained by those authors for a cluster of elements of radius $100 \mathrm{~km}$ and filling factor 0.2 . The plot shows clearly that contrast of large monolithic structures (isolated flux tubes) is lower than the contrast of smaller structures at disk center, while the opposite is observed off disk center. A sharp decrease from the center to the limb is observed for the isolated smallest structures, while smoother CLVs are observed for clusters of small elements. This finding indicates that at the limb clusters and large flux tubes are more likely to be observed rather than isolated small flux tubes and therefore weigh more in the estimation of the CLV of contrast derived by observations.

To investigate this effect we computed the average contrast at each position on the solar disk of the various models shown in the figure. To mimic selection effects associated with the application of intensity threshold criteria on images, average has been computed discarding contrast values smaller than 0.08 . The obtained CLV is represented by the red line in the plot of Fig. 7. The resulting CLV is flatter, especially at the limb, than the CLV of isolated flux tubes. Spruit (1976) investigated the CLV of maximum contrast obtained connecting the maxima of the CLVs of various flux tubes models. The author considered four classes of models and, in agreement with our findings, showed that for three classes of models the curves so obtained were flatter than 

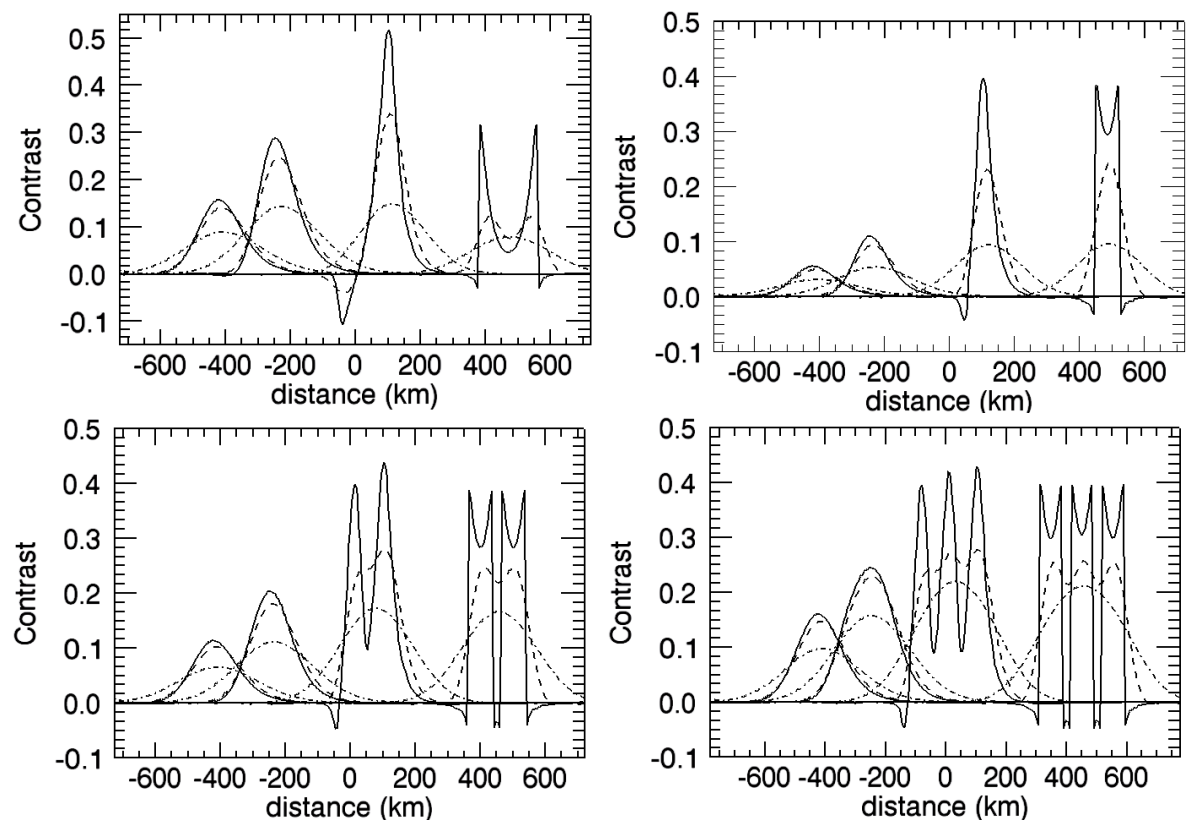

Fig. 6. Contrast profiles across flux tubes projected onto the plane of the sky for different resolutions and positions on the solar disk. Top left: isolated flux tube $175 \mathrm{~km}$ wide. Top right: isolated flux tube $70 \mathrm{~km}$ wide. Bottom left: two flux tubes $70 \mathrm{~km}$ wide $105 \mathrm{~km}$ apart. Bottom right: three flux tubes $70 \mathrm{~km}$ wide $105 \mathrm{~km}$ apart. Continuous line: resolution of simulations. Dashed line: resolution 0.1". Dot-dashed line: resolution $0.3^{\prime \prime}$. In each panel, from right to left: $\mu=1, \mu=0.9, \mu=0.6$, and $\mu=0.4$.

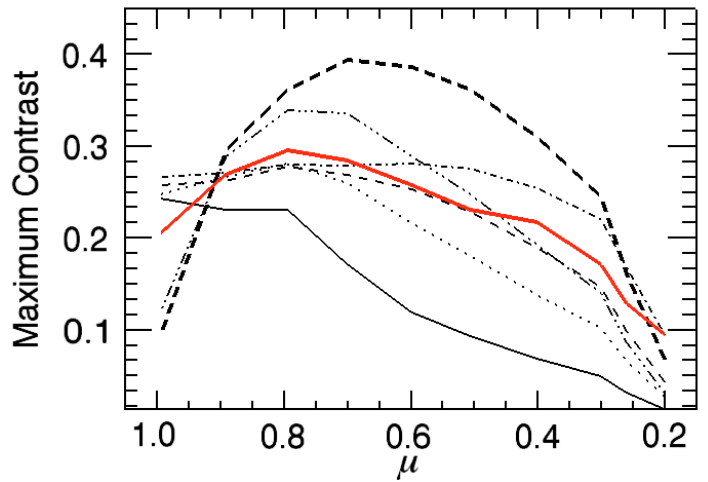

Fig. 7. CLV of maximum contrast for various flux tubes models and clusters at resolution $0.1^{\prime \prime}$. Continuous: single flux tube $70 \mathrm{~km}$ wide. Dotted: two flux tubes $70 \mathrm{~km}$ wide $105 \mathrm{~km}$ apart. Dashed: three flux tubes $70 \mathrm{~km}$ wide $105 \mathrm{~km}$ apart. Dot-dashed: five flux tubes $70 \mathrm{~km}$ wide $105 \mathrm{~km}$ apart. Dot-dot-dashed: single flux tube $175 \mathrm{~km}$ wide. Dashedthick: single flux tube $280 \mathrm{~km}$ wide. Initial Wilson depression is $150 \mathrm{~km}$ for all the models. Continuous red thick line: CLV of average maximum contrasts of all flux tubes configurations computed imposing a contrast threshold of 0.08 .

curves of individual flux tubes models, although the shapes of the curves were very different from the ones we have obtained.

As already noted for the contrast profiles, we found that the maxima of both maximum and average contrasts shift toward lower values of $\mu$ with the decrease of resolution for models in which dark lanes are large and dim. This effect is reduced in the case of aggregations of small flux tubes, for which dark lanes are less pronounced. Figure 8 shows for instance the CLV of maximum (black lines) and mean (red lines) contrast in the case of an isolated flux tube $175 \mathrm{~km}$ wide with initial Wilson depression $150 \mathrm{~km}$. Different line-styles represent different spatial resolutions. Squares and circles indicate the maxima of the curves, which shift toward the limb at the decrease of resolution. Here we define as mean contrast the average of contrast values larger than 0.02 . The plot also shows that, for a given spatial resolution, maximum and mean contrast values are correlated, i.e. the shapes of the curves are similar although the contrast values

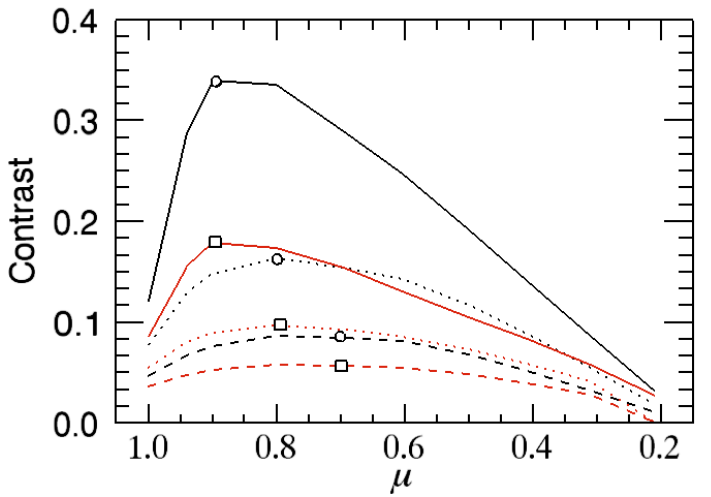

Fig. 8. CLV of maximum (black) and mean (red) contrast of a fluxtube $175 \mathrm{~km}$ wide. Spatial resolution is $0.1^{\prime \prime}$ (continuous), $0.3^{\prime \prime}$ (dotted) and $0.6^{\prime \prime}$ (dashed). Circles and squares indicate the maxima of the curves.

are different. We also notice that with the decrease of resolution the curves flatten and the decrease of contrast toward the limb is less abrupt. The dependence of the contrast-CLV on spatial resolution was previously reported by Okunev \& Kneer (2005), although a systematic shift of the peak with the decrease of resolution was not observed. This is most likely due to the lack of negative contrast area (dark lanes) in their simulations.

We also investigated the dependence of CLV of the mean contrast on the contrast threshold value applied. We found a change in the contrast values measured, but not substantial variation of the CLV shapes. Finally, we found that the contrastCLV is significantly dependent also on the opacity model, that is on the wavelength of observations. Figure 9 plots the CLV of maximum contrast of a cluster of three flux tubes $70 \mathrm{~km}$ wide and $105 \mathrm{~km}$ apart for the opacity models previously discussed. The largest changes in contrast occur at disk center (contrast decreases at wavelengths which form in the deepest layers), while at the extreme limb, contrast values are nearly independent of observation wavelength. As a consequence, the position of the peak of the CLV shifts from $\mu \approx 1$ at wavelengths which form 


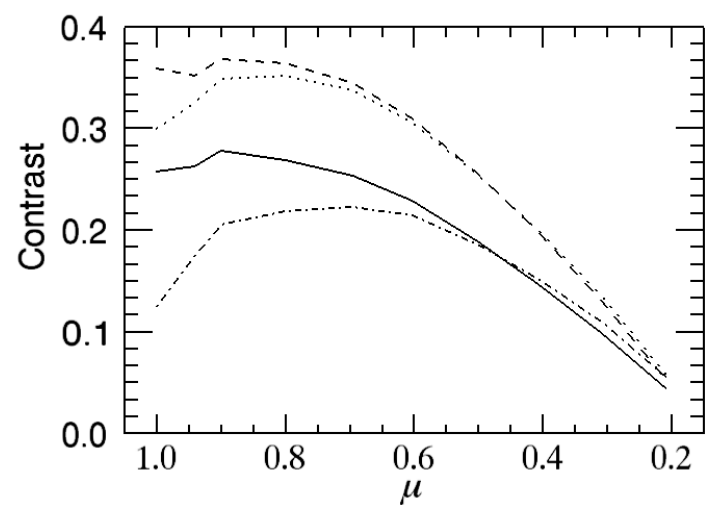

Fig. 9. CLV of maximum contrast of a cluster of three flux tubes $70 \mathrm{~km}$ wide $105 \mathrm{~km}$ apart observed with spatial resolution $0.1^{\prime \prime}$ in various continua. Continuous line: grey atmosphere. Dashed line: $\lambda=8000 \AA$. Dotted line: $\lambda=5000 \AA$. Dot-dashed line: $\lambda=16000 \AA$.

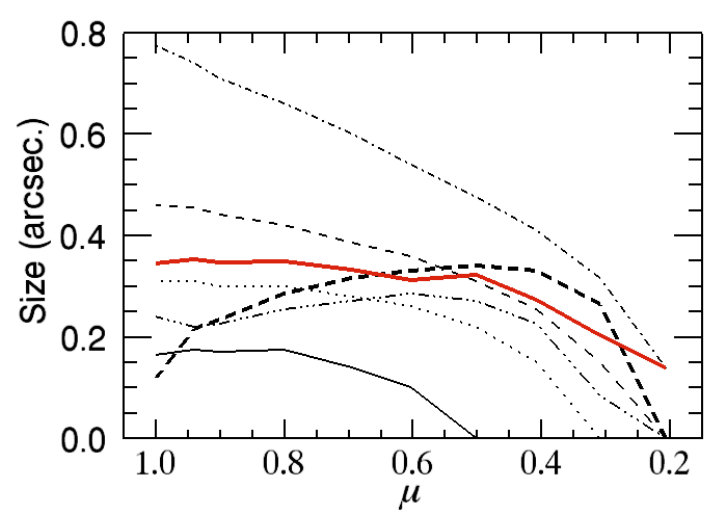

Fig. 10. CLV of size of different flux tubes models and clusters for contrast threshold value 0.08. Legend as in Fig. 7.

in the highest layers to lower values of $\mu$ at wavelengths which form in the deepest layers.

\section{Size and asymmetry}

We have investigated the geometric properties of isolated and clustered flux tubes. This analysis was performed on contrast profiles projected onto the plane of the sky and convolved with gaussian functions of different widths as explained in the previous paragraph.

We define the size of magnetic features as the largest distance between the points over which the contrast exceeds a given threshold. We find that the shape of the size-CLV is very dependent on the flux tube models investigated, as illustrated in Fig. 10. In general, CLVs of isolated or clusters of flux tubes which present marked double humps and dark lanes, show a minimum at approximately $\mu=0.9$ and a peak between $\mu=0.6$ and $\mu=0.4$. An example is given by dot-dot-dashed line in Fig. 10, which represents the size-CLV of a flux tube $175 \mathrm{~km}$ wide and initial Wilson depression $150 \mathrm{~km}$. The decrease of size from disk center to $\mu \approx 0.9$ is given by the smooth disappearance of the disk-centerward side wall and by the appearance of the dark lane, whose presence reduces the size of the "bright" area. The rise of size at intermediate lines-of-sights is due to the increase in width of that portion of the $\tau=1$ contour, which penetrates into the non magnetic atmosphere, as shown for instance in Fig. 3. The steep decrease at the extreme limb is due to both

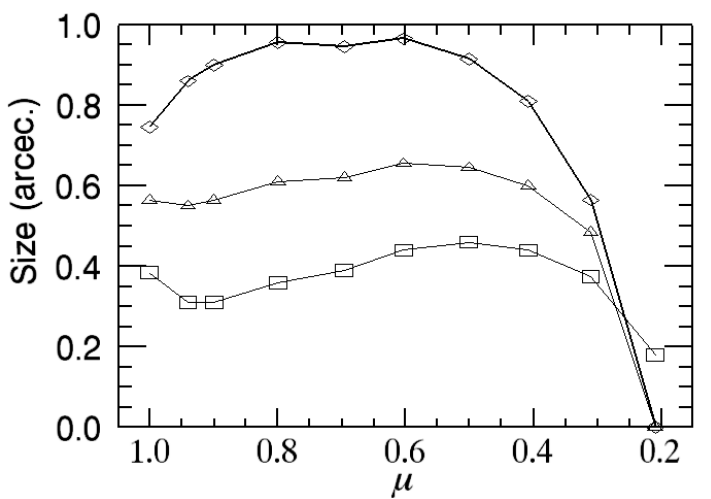

Fig. 11. CLV of size of a flux tube $175 \mathrm{~km}$ wide for various spatial resolutions. Square: $0.1^{\prime \prime}$. Triangle: $0.3^{\prime \prime}$. Diamond: $0.6^{\prime \prime}$. Contrast threshold value is 0.02 .

foreshortening, and a decrease in the size of the region with contrast values larger than the threshold applied. A CLV of this type was found by Steiner (2005) for a static flux tube $170 \mathrm{~km}$ wide and Wilson depression $150 \mathrm{~km}$. For models which do not present marked dark lanes or humps at disk center we found monotonic variations of size with position on the solar disk, as illustrated by most of the curves in Fig. 10. The other exception in that figure is the CLV of the $280 \mathrm{~km}$ wide flux tube, for which an increase from the center up to $\mu=0.4$ is observed. This is due to the fact that the contrast profile at disk center of this model is characterized by two positive contrast humps separated by a dark lane. According to our definition, the two humps are detected as two distinct features whose average size is the estimate of the size of the flux tube. This definition underestimates the size of the flux tube at disk center, thus generating the shape of the CLV illustrated. Finally, the red line is the average value of the sizes obtained by the models in the figure for each line-of-sight investigated. As for the contrast, the average size-CLV is flatter than the single models, especially at the limb.

The size-CLV curves also assume quite different shapes with variation in resolution. In general, the peak of the curve shifts toward disk center and the decrease of size toward the limb is steeper at lower spatial resolutions (Fig. 11). Moreover, while a decrease in the intensity threshold used to define structures corresponds an increase in the measured size, the CLV profiles remain similar to the ones obtained at lower resolution. An example of these effects is given in Fig. 12, which shows the size-CLV of a flux tube $70 \mathrm{~km}$ wide for four values of threshold contrast. It is worth noting that for high contrast threshold values or at poor spatial resolutions, small faint features are not detected.

As pointed out previously, projection onto the plane of the sky and reduction of resolution modify the shape of contrast profiles. To quantify these variations we analyzed the skewness of the profiles and defined as "asymmetry" the skewness of distribution of contrast values larger than a certain threshold. An example of the CLV of the asymmetries for threshold contrast value 0.001 and various spatial resolutions is illustrated in Fig. 13 in the case of a flux tube $175 \mathrm{~km}$ wide. We found that, in general, the skewness value is quite low, thus indicating that the contrast profiles are, overall, quite symmetric. The observed asymmetries are larger close to disk center and decrease toward the limb for the best spatial resolution investigated. Nevertheless, the decrease of resolution mostly affects vertical lines-of-sight, therefore for intermediate resolutions the asymmetries have a peak at approximately $0.4<\mu<0.6$. At the lowest resolution investigated the asymmetries decrease again 


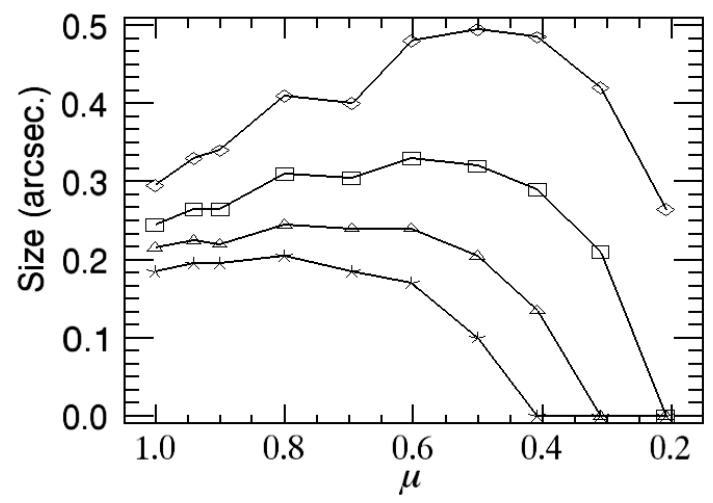

Fig. 12. CLV of size of a flux tube $70 \mathrm{~km}$ wide at resolution $0.1^{\prime \prime}$ for different contrast threshold values. Diamonds: 0.005. Square: 0.02. Triangle: 0.04. Asterisks: 0.06.

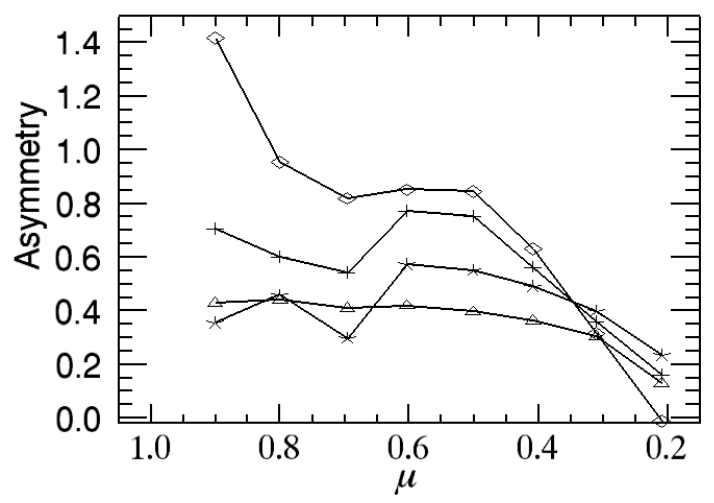

Fig. 13. CLV of skewness at various spatial resolutions for a flux tube $175 \mathrm{~km}$ wide. Diamonds: resolution of simulation. Plus: $r=0.1^{\prime \prime}$. Asterisks: $r=0.3^{\prime \prime}$. Triangles: $r=0.6^{\prime \prime}$.

monotonically from disk center to the limb. It is also worth noticing that at $\mu<0.4$ profiles are nearly symmetric. This is not in disagreement with findings of previous authors, who reported an increase in the limb ward tail with the inclination of the lineof-sight (e.g. Steiner 2005; Pizzo et al. 1993b; Deinzer et al. 1984), since here we are considering profiles project onto the plane of the sky and, as previously shown, projection mostly affects the extreme limb. Moreover, this is in agreement with Berger et al. (2007), who found that observed average contrast profiles at $\mu=0.4$ are more symmetric than average profiles at $\mu=0.6$. We also found that the value of the asymmetries defined in this way increases with the size of isolated flux tubes and with the number of tubes in a cluster. Finally, it is also important to notice that the skewness poorly describes the asymmetries of distributions which have multiple peaks and is therefore not a good indicator of the asymmetries for profiles of isolated large flux tubes at $\mu>0.9$, or in the cases for which the profiles of clusters of tubes show multi-peaks.

\section{Contrast-size relation}

Scatter plots of measured contrast against magnetic feature size presented in the literature usually show a large dispersion. Fits to these points have shown no trend (Berger et al. 1995; Wiehr et al. 2004; Sánchez Almeida et al. 2008) or a slight increase (Bovelet \& Wiehr 2003; Hirzberger \& Wiehr 2005; Berger et al. 2007) of contrast with magnetic feature size. In particular, Berger et al. (2007) showed that the contrast-size dependence of magnetic
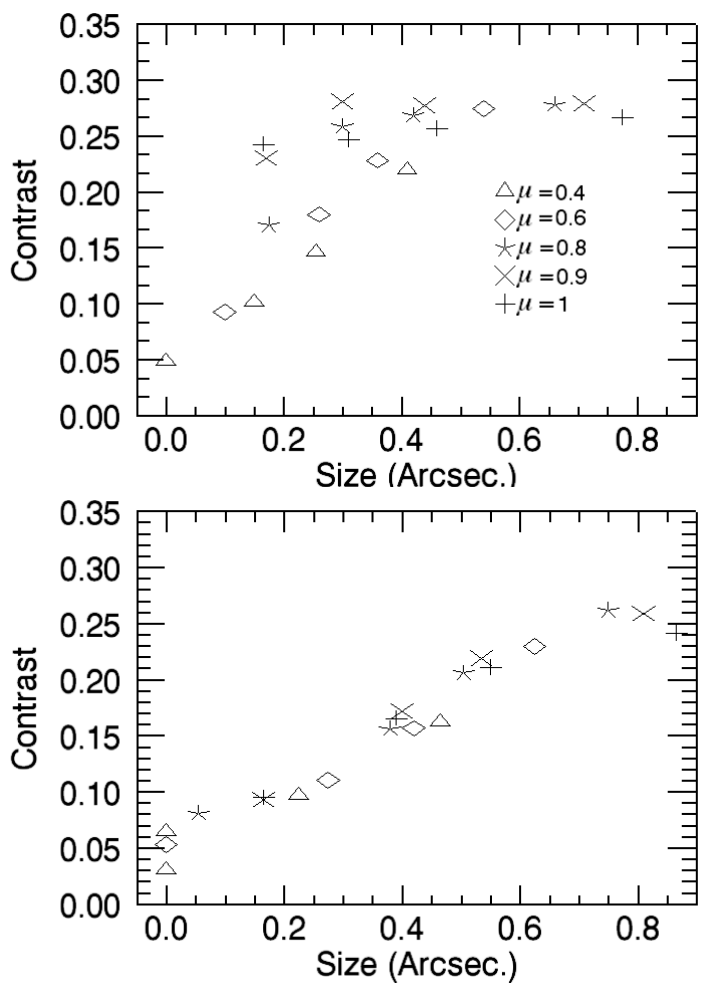

Fig. 14. Size versus contrast of four clusters of flux tubes at various positions on the solar disk. Top: spatial resolution 0.1". Bottom: spatial resolution $0.3^{\prime \prime}$.

features for various positions on the solar disk can be fitted by parallel straight lines. A closer inspection of their Fig. 5 reveals that, especially for off-disk-center features, the increase in contrast is sharper at the smallest areas, while no trend is observed at the largest areas. This is in agreement with Bovelet \& Wiehr (2003) and Hirzberger \& Wiehr (2005) and also qualitatively with results obtained by analyses of full-disk images (see Ermolli et al. 2007, and references therein).

In order to investigate whether spatial resolution of observations could explain such discrepancies, we studied the contrastsize relation of various clusters of flux tubes. Figure 14 shows the contrast-size relation for an isolated flux tube $70 \mathrm{~km}$ wide and for clusters of two, three and five tubes $70 \mathrm{~km}$ wide and $105 \mathrm{~km}$ apart at various positions on the solar disk and for spatial resolution $0.1^{\prime \prime}$ (top) and $0.3^{\prime \prime}$ (bottom). For vertical directions ( $\mu=1$ and $\mu=0.9$ ) and for resolution $0.1^{\prime \prime}$ contrast does not vary significantly with features size. At shallower lines-of-sight, the contrast increases with size. For $\mu=0.8$ and $\mu=0.6$ size dependence of contrast is larger for the smallest sizes while there is no dependence at the largest sizes.

The increase in the contrast with size at inclined lines-ofsight is due to the reduced attenuation of radiation in the largest clusters. Moreover, since for each inclination, there is a finite number of flux tubes that radiation can cross (see Sect. 4), there is a size threshold beyond which contrast does not vary with size. This size threshold increases with the decrease of $\mu$. An increase in contrast with size is observed even at vertical lines-of-sight with the reduction of spatial resolution, as illustrated in bottom panel of Fig.14. This is due to the larger reduction in contrast value due to smoothing on the smallest features (see Sect. 4). Bottom plot shows that at each position on the solar disk, data can be well fitted with straight lines, as obtained in observational data by Berger et al. (2007). 


\section{Summary and conclusions}

We have investigated thermal, photometric and geometric properties of various configurations of clusters of magnetic flux tubes by two dimensional, static numerical simulations. The main results, obtained by the analyses of tubes with Wilson depression $60 \mathrm{~km}$ when in RE, are the following.

At depths for which radiative heating is efficient, the temperature stratification within and around flux tubes is function not only of the physical properties of the flux tubes, but also of the tube number. In fact we found that the temperature inside the flux tubes increases with the number of adjacent tubes in the optically thin part of the domain and decreases at $0<\log (\tau)<1$. At larger values of optical depth temperature increases again with the filling factor. The area around and between the tubes is cooled at $0.5<\tau<2$, while is heated at greater depths.

Since the temperature stratification determines photometric properties, contrast profiles of flux tubes also vary in clusters. At disk center contrast increases with the number of flux tubes. Moreover regions in between the tubes are characterized by negative contrast, which is a signature of the decrease of temperature, generated by the radiative cooling, in these areas. These dark features gradually disappear with the increase in the inclination of the line-of-sight. We found that for each cluster model there always exist an inclination beyond which clusters appear as compact monolithic features, as if they were isolated flux tubes. At these inclinations, the contrast of a cluster is larger than the contrast of a single flux tube element, and smaller than the contrast of an isolated tube with the same size and Wilson depression of the cluster.

As for isolated flux tubes, off-disk-center contrast profiles of clusters are very asymmetric, with long tails toward the limb and dark lanes at disk-center side. Nevertheless, projection onto the plane of the sky and the decrease of the spatial resolution (due for instance to both instrumental and atmospheric degradation), largely makes profiles more symmetric. This finding is in agreement with measurements off-disk-center of G-band bright points contrast profiles by Berger et al. (2007). These authors found quite symmetric profiles at all disk positions investigated. In both our models (Fig. 13) and observations, profiles at $\mu=0.6$ are more symmetric than profiles at $\mu=0.4$. Reduction of spatial resolution also decreases the depth and size of dark rings and dark lanes, which disappear in the worst resolution cases investigated $\left(0.3^{\prime \prime}\right)$. Moreover, dark lanes are not ubiquitous features of flux tubes. Since these are signatures of cooler regions within and around the tubes, they are very faint or absent in tubes models with larger radiative heating (see also models presented in Steiner 2005 or Pizzo et al. 1993b), or can be observed in some wavelengths and not in others. This helps explain why Berger et al. (2007) did not find dark lanes in all the contrast profiles they observed off disk center.

CLVs of properties of magnetic bright features are also affected by clustering and reduction of resolution. The contrastCLV is for instance steeper for isolated flux tubes than it is for clusters of tubes. Since most of feature identification techniques on images are based on intensity threshold, isolated small (and therefore less brilliant) features are less likely to be detected. Measurements at the limb are therefore biased toward larger tubes and clusters and the resulting CLV is flatter than the one obtained for single structures (see Fig. 7). For the same reasons, the CLV of sizes is also flatter for tube clusters (see Fig. 10). This would explain CLV shapes obtained by recent observations at the extreme limb (Hirzberger \& Wiehr 2005). Reduction of resolution also shifts the peak of the contrast-CLV toward lower values of $\mu$, especially for tubes models with marked dark lanes, and makes the CLVs flatter (see Fig. 8). In this context, we notice that Berger et al. (2007), who analyzed better resolution images with respect to other authors, found the peak of the CLV of contrast to occur closer to the disk center with respect to some previous works (see Berger et al. 2007, and references therein).

Finally, we have investigated the size-contrast relation for clusters of various numbers and spatial resolutions. We found a slight increase in contrast with size at disk center, which has to be partially ascribed to the increase in temperature with the number of adjacent tubes and partially to the larger effects of smoothing on smaller features. Increase of contrast with size (and therefore with the number of flux tubes) is found for inclined lines-of-sight due to the reduced opacity inside the tubes. Since for a given inclination of the line-of-sight only a finite number of tubes is crossed by radiation (see Sect. 4), there is a size threshold beyond which contrast does not vary. This threshold increases with the inclination of the line-of-sight. With the decrease of spatial resolution, even for vertical lines-of-sight the contrast increases with size, the threshold size value increases, and the contrast-size curves obtained at various lines-of-sight can be fitted by straight parallel lines. These findings are in agreement with recent contrast-size measurements obtained on both high (Berger et al. 2007) and medium (Ermolli et al. 2007) resolution images.

Results presented in this work suggest that measurements of properties of magnetic bright features are significantly influenced by spatial resolution, image analysis techniques, and the wavelength of observation. Geometric properties of contrast profiles, such as size and asymmetries, are particularly affected. These findings can partially explain the discrepancies presented in the literature. Moreover, some observational results can be better interpreted by considering observed bright magnetic features as aggregation of smaller elements, rather than a monolithic entity.

The results presented in this work should be properly taken into account when trying to derive physical properties of magnetic flux tubes by observations.

Acknowledgements. The authors are grateful to V. Penza for providing the opacity and atmosphere models and to the referee for the helpful review of the manuscript. S. C. acknowledges the OAR grant, which is based on the CVS project supported by Regione Lazio. S. C also thanks I. Ermolli for useful discussions and support.

\section{References}

Auffret, H., \& Muller, R. 1991, A\&A, 246, 264

Berger, T. E., Schrijver, C. J., Shine, R. A., et al. 1995, ApJ, 454, 531

Berger, T. E., Rouppe van der Voort, L., Löfdhal, M., et al. 2004, A\&A, 428, 613

Berger, T. E., Rouppe van der Voort, L., \& Löfdhal, M. 2007, ApJ, 661, 1272

Bovelet, B., \& Wiehr, E. 2003, A\&A, 412, 249

Caccin, B., \& Severino, G. 1979, ApJ, 232, 297

Cannon, C. J. 1970, ApJ, 161, 255

Carlson, B. G. 1963, in Methods in Computational Physics, 1, ed. B. Alder, S. Fernbach, \& M. Rotenberg

Carlsson, M., Stein, R. F., Nordlund, Å., \& Scharmer, G. B. 2004, A\&A, 610, 137

Criscuoli, S. 2007, Ph.D. Thesis, University of Rome "TorVergata"

Deinzer, W., Hensler, G., Schüssler, M., \& Weisshaar, E. 1984, A\&A, 139, 435 De Pontieu, B., Carlsson, M., Stein, R., et al. 2006, ApJ, 646, 1405

Ermolli, I., Criscuoli, S., Centrone, M., Giorgi, F., \& Penza, V. 2007, ApJ, 465, 305

Fabiani-Bendicho, P., Kneer, F., \& Trujillo-Bueno, J. 1992, A\&A, 264, 229

Giordano, S., Berrilli, F., Del Moro, D., \& Penza, V. 2008, A\&A, 489, 747

Grevesse, N., Noels, A., \& Sauval, A. J. 1993, A\&A, 271, 587

Hasan, S. S., Kalkofen, W., \& Steiner, O. 1999, in Solar polarization, ed. K. N

Nagendra, \& J. O. Stenflo (Kluwer Academic Publishers), ASSL, 243, 409 
Hasan, S. S., van Ballegooijen, A. A., Kalkofen, W., \& Steiner, O. 2005, ApJ, 631,1270

Hirzberger, J., \& Wiehr, E. 2005, A\&A, 438, 1059

Ishikawa, R., Tsuneta, S., Kiakoshi, Y., et al. 2007, A\&A, 472, 911

Karpinsky, V. N., \& Okunev, O. V. 1998, Sol. Phys., 183, 277

Keller, C. U., Schüssler, M., Vögler, A., \& Zakharov, V. 2004, A\&A, 607, L59

Kneer, F., \& Trujillo-Bueno, J. 1987, A\&A, 183, 91

Knölker, M., \& Schüssler, M. 1988, A\&A, 202, 275

Knölker, M., Schüssler, M., \& Weisshaar, E. 1988, A\&A, 194, 257

Kunasz, P., \& Auer, L. 1988, J. Quantum Spectrosc. Radiat. Transf., 39, 67

Kurucz, R. L. 1994, CD-ROM No. 19

Lites, B. W., Scharmer, G. B., Berger, T. E., \& Title, A. M. 2004, Sol. Phys., 221, 65

Nisenson, P., van Ballegooijen, A. A., de Wijn, A. G., \& Sütterlin, P. 2003, ApJ, 587,458

Okunev, O. V., \& Kneer, F. 2004, A\&A, 425, 321

Okunev, O. V., \& Kneer, F. 2005, A\&A, 439, 323
Pizzo, V. J., MacGregor, K. B., \& Kunasz, P. B. 1993a, ApJ, 404, 788 Pizzo, V. J., MacGregor, K. B., \& Kunasz, P. B. 1993b, ApJ, 413, 764 Rouppe van der Voort, L. H. M., Hansteen, V. H., Carlsson, M., et al. 2005, A\&A, 435, 327

Sánchez Almeida, J., Márquez, Bonet, J. A., Domínguez Cerdena, I., \& Muller, R. 2008 [arXiv:astro-ph/0405515v1]

Sánchez Cuberes, M., Vázquez, M., Bonet, J. A., \& Sobotka, M. 2002, ApJ, 570, 886

Spruit, H. C. 1976, Sol. Phys., 50, 269

Steiner, O., \& Stenflo, J. O. in IAU Symp. 138, ed. J. O. Stenflo, 131 Steiner, O. 2005, A\&A, 430, 691

Stix, M. 2002, The Sun: An Introduction, 2nd edn. (Berlin: Springer) Sütterlin, P., Wiehr, E., \& Stellmacher, G. 1999, Sol. Phys., 189, 57

Uitenbroek, H. 1998, ApJ, 498, 427

Uitenbroek, H., \& Tritschler, A. 2006, ApJ, 639, 525

Vögler, A. 2004, ApJ, 421, 755

Wiehr, E., Bovelet, B., \& Hirzberger, J. 2004, A\&A, 422, 63 